\title{
MUSICOLOCIA Y TICS
}

\section{LAS MUJERES EN EL SONIDO:}

ANDROCENTRISMO EN LA INDUSTRIA MUSICAL

WOMEN IN AUDIO: ANDROCENTRISM IN THE MUSIC INDUSTRY

M. Francisca Barrios-Ruano

Ana M. Muñoz-Muñoz 
RECIBIDO: $29 / 04 / 2021$

ACEPTADO: $15 / 05 / 2021$

\section{RESUMEN}

En este trabajo se plantea teóricamente la existencia de androcentrismo en los puestos de producción e ingeniería del sonido en la industria musical en los que se perpetúan los modelos estereotipados de distribución laboral, donde las mujeres desempeñan o son relegadas a roles feminizados y, principalmente, están alejadas de las posiciones que conllevan autoridad. Para ello, se ha analizado el estado de los estudios musicológicos en relación con los estudios de género y la crítica feminista, estudiando la interrelación de las mujeres con la industria musical y con la tecnología del sonido musical, examinando la representación de las mismas en diversos campos profesionales y observando la construcción de redes de trabajo de mujeres en el sonido. El análisis de estos estudios sugiere que las mujeres, que se desarrollan profesionalmente en el campo del tratamiento técnico del sonido en la industria musical, podrían dotar a su labor profesional de una nueva significación de género al cuestionar el capital social y los cánones establecidos.

\section{PALABRAS CLAVE}

\section{Mujeres en el sonido, Musicología feminista, Industria musical, Redes trabajo,}

\section{Estudios de las Mujeres}

\section{A B S T R A C T}

$\mathrm{n}$ this paper the existence of androcentrism is theoretically proposed in sound production and engineering positions in the music industry in which stereotypical models of labor distribution are perpetuated, where women play or are relegated to feminized roles and, mainly, they are far from positions that carry authority. For this, the state of musicological studies in relation to gender studies and feminist criticism has been analyzed, studying the interrelation of women with the music industry and with the technology of musical sound, examining their representation in various professional fields and observing the construction of women's networks in sound. The analysis of these studies suggests that women, who develop professionally in the field of technical sound treatment in the music industry, could give their professional work a new gender significance by questioning social capital and established canons.

\section{KEYWORDS}




\section{ANTECEDENTES DE LOS ESTUDIOS}

\section{SOBRE MUJERES Y MÚSICA}

El campo de los estudios musicales ha sido menos permeable a la crítica feminista y a los estudios de género que otras disciplinas académicas tales como las humanísticas, las ciencias de la salud, las ciencias sociales o el cine (Viñuela, 2004; Cabedo, 2009). Aunque existen estudios pioneros, como el publicado en 1948 de Sophie Drinker titulado Music and Women: the Story of Women in their Relation to Music, la musicología no incorporó la crítica a su tradición androcéntrica hasta prácticamente los años 80 del pasado siglo (Ramos López, 2003 y 2010 ).

En la década de 1980 se desarrolló, primero en EE.UU. y posteriormente en Europa, una ingente labor de investigación para recuperar figuras tanto de compositoras como de intérpretes que habían sido sepultadas bajo el entramado ideológico patriarcal de la Historia canónica de la Música. Este marco de estudio, denominado historia contributiva (Viñuela, 2004) o historia compensatoria ${ }^{1}$ (Soler, 2018) no llegó a replantearse las categorías historiográficas ni llegó a analizar "en profundidad las razones sociales y políticas que llevaron a la invisibilidad de las mujeres músicas" (Viñuela, 2004 , p. 21); en cambio, se centró en la búsqueda de datos de la obra, profesionalización y vida de las mujeres en relación con sus actividades musicales, de las mecenas y patrocinadoras, de las orquestas femeninas, de la edición de partituras de las mujeres músicas... (Cascudo García-Villaraco, 1998).

1 "Lamamos historia compensatoria a aquella que ha redescubierto a la mujer en todas las facetas musicales desde la Antigüedad hasta nuestros días, desarrollando su labor como compositoras, intérpretes, mecenas, docentes, con una recepción clara" (Soler Campo, 2018, p. 91).
En este marco cabría mencionar trabajos: Women in Music: A Bibliography de Don L. Hixon y Don Hennesse (1975); la International Encyclopedia of women composers de Aaron Cohen (1981); Donne in Musica de Patricia Adkins Chiti (1982); la colección de artículos de Jane Bowers y Judith Tick (1987) Women Making Music: The Western Art Tradition y la Historical Anthology of Music by Women de James R. Briscoe (1987), o el New Grove Dictionary of Women Composers de Julie Anne Sadie y Rhian Samuel (1994) (Ramos López, 2003; Piñero, 2003).

Además, en España señalaríamos el apéndice de compositoras españolas que adjuntó en 1995 María Luisa Ozaita al libro traducido de Patricia Adkins, diversos trabajos de Josemi Lorenzo Arribas (1998) como Las mujeres y la música: una relación disonante o el trabajo publicado por el Centro de Documentación de Música y Danza (2008): Compositoras españolas, la creación femenina desde la Edad Media hasta la actualidad. Todos estos estudios pusieron de manifiesto la existencia, hasta entonces desconocida u olvidada, de mujeres en todos los ámbitos y etapas musicales.

\section{NUEVA MUSICOLOGÍA: MUSICOLOGÍA FEMINISTA}

Aunque "la descripción de la participación de las mujeres en la música a lo largo de la historia de la música occidental es necesaria para construir unos antecedentes cuya existencia hasta hace muy poco ha sido negada" (Zavala, 2012, p. 123), no fue hasta comienzos de los años 90 cuando se empezaron a publicar distintas investigaciones en las que también se cuestionaban los paradigmas tradicionales de los estudios musicológicos. Esta nueva corrien- 
te, que se caracteriza por la heterogeneidad, se denomina Nueva Musicología ${ }^{2}$. Se pregunta acerca de conceptos como el de la música en sí, el del canon musical, el de la autonomía de la obra musical, el de la idea de objetividad y neutralidad, la noción de lo extramusical, el contenido semiótico de la música e, igualmente, amplía el campo de estudio hacia músicas no occidentales y músicas populares; rechaza, a su vez, el positivismo y formalismo y está influenciada por la pluralidad del pensamiento posmodernista que critica el ideal de la objetividad de la razón moderna y que "deconstruye el esencialismo de conceptos como raza, etnia, clase, género, edad..." (Ramos López, 2003, p. 37). La Nueva Musicología engloba diferentes líneas de pensamiento como son el Feminismo, la Narratología, La Hermenéutica, la Sociomusicología y la Crítica postadorniana. Es por lo que Pilar Ramos López (2003) sugiere que podría ser más acertado referirse a Musicologías Posmodernas en lugar de a Nueva Musicología.

De este modo, hablamos de la musicología feminista que se ocupa, entre otros campos, de: analizar la existencia de un lenguaje musical femenino propio, subrayar cómo opera el sistema del patriarcado en todas las parcelas musicales, poner en valor las diferentes condiciones en las que las mujeres desarrollan las actividades musicales respecto a los hombres, visibilizar la contribución de las mujeres a las diversas ramas de la música, clarificar la exclusión de las mujeres músicas en las historias de la música, investigar las construcciones de identidades de género a través del discurso musical, estudiar las relaciones entre género y organología, formas musicales, análisis musical, tímbrica o tesitura vocal, identificar la atribución de significados musicales y educación musical, considerar los constructos de género en la música y en sus instituciones, analizar estereotipos de género en las letras de obras musicales y en la elección de estudios musicales, identificar los roles asignados a los personajes de las obras escénicas musicales cantadas y bailadas, señalar la dificultad de encontrar a mujeres alejadas de las posiciones que conllevan un mayor estado de poder en la música, examinar los códigos semióticos utilizados en la teoría y en el lenguaje musical; en definitiva, deconstruir la idea de la música como lenguaje autónomo, universal independiente tanto de

2 Témino acuñado por Lawrence Kramer en 1990 Niñuela, 2003, p. 11). su contexto histórico como de las personas que lo desarrollan (Die Goyanes, 1998; Viñuela, 2003 y 2004; Cabedo, 2009; Ramos López, 2013; Soler, 2018).

De entre los numerosos estudios que se han realizado desde estas líneas de investigación destacan: Feminine Endings; Music, Gender and Sexuality de Susan McClary en 1991, Gender and the Musical Canon (Citron, 1993), Música, Género y Educación (Green, 2001) y el trabajo editado Susan C. Cook y Judy S. Tsou en 1994 titulado Cecilia Reclaimed. Feminist Perspectives on Gender and Music.

Igualmente, en España destacan, especialmente, los títulos: Música y Mujeres. Género y Poder (Manchado, 1998), Feminismo y música. Introducción crítica (Ramos López, 2003), La perspectiva de género y la música popular: dos nuevos retos para la musicología (Viñuela, 2004).

Si hasta este momento el foco de atención de los estudios musicológicos se había centrado fundamentalmente en el análisis de la obra musical ajena a cualquier contexto político-social, será a partir de la última década del siglo XX cuando este vire hacia zonas fronterizas, ampliando los campos de estudio a, por ejemplo, las condiciones de producción, difusión, recepción de la música en general; deteniéndose también en la música popular urbana y, más en concreto, en el estudio de la música popular con perspectiva de género (Ramos López, 2010; Viñuela, 2004).

\section{LAS MUJERES EN LA INDUSTRIA MUSICAL}

Será en el siglo XX cuando la música popular adquiera mayor repercusión y difusión debido, entre otros factores, al desarrollo tecnológico de los medios de producción, grabación, reproducción y comunicación del sonido.

"La música pasa por diferentes procesos y etapas relacionadas con la propia composición, la producción, la edición, distribución etc. Son muchas, por tanto, las etapas y personas que intervienen en el proceso productivo de la música popular" (Martínez Escribano, 2011 ). Durante muchos años las mujeres que profesionalmente querían desarrollarse en este campo encontraban serias dificultades para abarcar todas esas etapas y escenas musicales puesto que, normalmente, se veían avocadas a des- 
empeñar roles feminizados, principalmente como cantantes, encargadas de marketing, publicidad... (Green, 2001; Bilbao, 2015; Lores, 2017). Este hecho es debido a que la música, como cualquier otra manifestación cultural, es susceptible de generar y transmitir construcciones de género y durante mucho tiempo, incluso en la actualidad, ha estado controlada por una clase hegemónica que ha impuesto su ideología dominante que censura determinados comportamientos en las mujeres, a la vez que duda de las aptitudes intelectuales para realizar determinadas actividades musicales (Ramos López, 2003). Es lo que sostiene Lucy Green al afirmar que "la música evoca la masculinidad, una mente masculina, a un hombre tras la música; y esto se ha considerado tan normal y aceptable que ni siquiera nos percatamos de su vigencia hasta que ocurre algo que la quiebra" (2001, p. 112).

La discriminación de género afecta a las mujeres en prácticamente cualquier ámbito laboral que se analice. La falta de paridad, la desigualdad de oportunidades, la brecha salarial, el acoso y/o abuso sexual en el trabajo, el escaso reconocimiento profesional, la invisibilidad, el techo de cristal y/o los estereotipos de género, son realidades que las mujeres profesionales han vivido y viven en sus prácticas laborales en general; lamentablemente, el mundo vinculado al sector musical tampoco es ajeno a ello.

La música es, posiblemente, la expresión cultural que presenta mayor grado de industrialización. La música se ha convertido en industria musical. En esta podríamos distinguir varios niveles en función del entramado de profesionales que agrupan y del trabajo que desarrollan dentro de la misma. Así en un primer nivel nos encontraríamos con las personas vinculadas al propio proceso creativo musical como compositoras/es, cantantes, instrumentistas, letristas o arreglistas. En un segundo nivel, se situarían las personas que trabajan en empresas cuyo objetivo es crear, promocionar y vender música grabada o en directo como las personas especialistas en sonido (ingenieras/os y técnicas/os de sonido), las personas encargadas de la producción musical, del management, los estudios de grabación, las compañías discográficas, las editoriales musicales... El último nivel, correspondería a aquellas personas responsables de difundir y distribuir la música creada como las responsables de la prensa musical, de la promoción de espectáculos, las que trabajan en las salas de conciertos con actuaciones en vivo o en las superficies de venta al público (Mañó y Bori, 2016). En general, podríamos decir que "la industria musical en su conjunto vive de la creación y la explotación de la propiedad intelectual de la música" (Wikström, 2014, p. 10).

La música ha pasado a convertirse en un sector estratégico de la economía mundial. Tanto si nos fijamos en el sector de la industria de la música que se encarga de la explotación discográfica 0 en el de servicios musicales basados en acceso o en el que se ocupa de las licencias musicales o en el de la música en directo, el impacto directo de todos ellos en la economía es muy significativo. Eso, sin mencionar otros sectores como podrían ser el merchandising musical, el de la fabricación de equipos de sonido, de software, de instrumentos... que, si bien están íntimamente ligados con la industria musical, no se consideran de manera estricta pertenecientes a ella.

Así, a finales del año 2013 la empresa de servicios musicales basada en acceso, Spotify, generó algo más de 1.000 millones de dólares de beneficio las para personas propietarias de derechos de todo el mundo (Wikström, 2014). En 2014, el valor de las ventas de álbumes en el primer país por volumen de ventas, EE.UU., alcanzó casi los 4.900 millones de dólares (PromociónMusical.es, s.f.). La red de asociaciones de salas de música europea Live DMA (2020), que representa a casi 2.600 locales de música en directo en Europa señaló que tras el análisis de los datos de facturación correspondientes al año 2017, estas salas y clubs de música en vivo generaron 1.800 millones de euros directos de facturación, sin mencionar los numerosos efectos secundarios, no solo económicos sino también culturales y de empleo, que positivamente repercutieron en las localidades donde están situados dichos locales.

En el estado español, la industria musical ha aumentado durante el primer semestre de 2020 sus cifras globales de ventas (físicas y digitales) en un 3,99\% respecto al mismo periodo del año anterior, alcanzando los 145 millones de euros (Fuente, 2020). Además, según la Asociación de Promotores Musicales (2020) el año 2019, el último del que se disponen de cifras, ha sido el que ha alcanzado una mayor facturación en espectáculos y conciertos de música en directo con más de 382 millones de euros y con un impacto estimado del 3,8\% en 
el PIB español.

En esta mercantilización y masificación del consumo del arte, en esta llamada convergencia de la cultura, cabría preguntarse qué posición ocupan las mujeres en esta industria. Esta cuestión era planteada por Banks y Millestone en su trabajo Individualization, gender and cultural work:

In the 'new' economy the virtues of creative and cultural industry production are widely promoted and idealized. For women, set free from their 'feudal chains', the 'cool creative and egalitarian' cultural economy - particularly in areas such new media, music, design and fashion - appears to offer paths to workplace freedom. But is this really so? $?^{3}(2011$, p. 73).

Las mujeres, sin embargo, siguen presentando unas trayectorias profesionales divergentes con respecto a los hombres (Martínez Berriel, 2011 ). Las estadísticas, que diversos estudios han realizado, ponen de manifiesto que las mujeres que desempeñan su labor profesional dentro de la industria musical están infrarrepresentadas en la mayoría de sus áreas (Mujeres y Música, 2021; Smith, 2020; Cruz, 2019; Prior et al., 2018). Se estima que, en las profesiones musicales de la industria musical, la presencia femenina solo alcanza de media un $30 \%$ en las mismas (Marinas, 2019). Varios motivos pueden ser la causa de esta baja presencia de mujeres en los sectores musicales Gaston-Bird, 2020; Ispizua, 2020; Marinas, 2019; Keychange, 2018; Savage, 2012; Haruch, 2010; Adkins, 2006), y que no serían muy dispares a los ya estudiados en otros campos profesionales, como los siguientes:

- Las posiciones de poder del sector están en su mayoría ocupadas por hombres y por tanto perpetúan la jerarquía de poder masculino que dificulta el que las mujeres accedan a puestos directivos y a toma de decisiones.

3 En la "nueva" economía, las virtudes de la producción de la industria creativa y cultural son ampliamente promovidas e idealizadas. Para las mujeres, liberadas de sus "cadenas feudales", la economía cultural "cool, creativa e igualitania" — particularmente en áreas como los nuevos medios, la música, el diseño y la moda — - parece ofrecer caminos hacia la libertad laboral. Pero, ટ̇es esto realmente así? (Esta y todas las demás traducciones son realizadas por la autora).
- Las diferentes exigencias personales y profesionales que se esperan de las mujeres y no de los hombres derivadas de la maternidad o el cuidado familiar.

- Estereotipos de género como la creencia de menores habilidades creativas y/o técnicas en las mujeres y tener que demostrarlas continuamente frente a otros aspirantes masculinos o el asociar el desempeño de determinados trabajos frente a otros solo por el hecho de ser mujer.

- La falta de referentes profesionales de mujeres en donde las jóvenes generaciones puedan guiarse, fijarse e inspirarse y que les permitan escoger unos estudios y unas carreras musicales específicas.

Ambientes de trabajo poco empáticos con las mujeres, donde una masa laboral dominante masculina actúa como barrera para las mujeres profesionales de la industria de la música, quienes a menudo se enfrentan a equipos de trabajo solamente compuestos por hombres. Este factor es especialmente significativo en determinados campos del sector musical como en los estudios de grabación o en las giras de conciertos.

En definitiva, se estima que la industria musical perpetúa el modelo estereotipado de la distribución laboral, en donde las mujeres desempeñan o son relegadas a roles feminizados $y$, principalmente, están alejadas de las posiciones que conllevan poder (Lores, 2017; Quiñones, 2019, Marinas, 2019), reproduciéndose en este sector lo señalado por Die Goyanes (1998), Ramos López (2003), Viñuela (2004) y Soler (2018) respecto a lo que ocurre en el plano puramente musical con las compositoras, directoras de orquesta o intérpretes.

\section{LAS MUJERES EN EL SONIDO}

"Así, podemos encontrar muchas mujeres en áreas de marketing y comunicación, pero pocas en áreas técnicas, prensa o producción" (Marinas, 2019, p. 18).

Diversos estudios sugieren que dentro de la industria musical, en donde ya de por sí en todos sus campos existe una brecha de género, las profesiones que para el desempeño de su ejercicio tienen una mayor relación con los campos tradicionalmente asociados al mundo masculino, los de las ciencias, las tecnologías, 
las ingenierías y las matemáticas, también denominadas disciplinas STEM si utilizamos el acrónimo en inglés de estas disciplinas: Science, Technology, Engineering and Math, y los que implican un gran grado de creatividad, presentan a su vez una menor representación de mujeres que en otros campos de la industria. Estaríamos hablando de, por ejemplo, las profesiones relacionadas con el tratamiento técnico del sonido (Gaston-Bird, 2020; Martínez Berriel, 201 1; González González, 1999).

\footnotetext{
"I thought, 'Nah, that's not for me' Why did I think that? It's a combination of not seeing women doing it. It feeds on itself... There's an assumption that femaleness is not attached to engineering" 4 (Kathryn, 2019, como se citó en Gaston-Bird, 2020, pp. 75-76).
}

En general, los campos profesionales más específicamente relacionados con el tratamiento técnico del sonido (personas especialistas en sonido -ingenieras/os y técnicas/os de sonido-, las personas encargadas de la producción musical, del sonido en radio, películas, televisión, videojuegos...) presentan una menor representación femenina y un mayor sesgo androcéntrico (Mathew et al, 2016; Prior et al., 2018; Hopkins, 2019).

4 "Pensé, ỉah, eso no es para mí! ¿̇Por qué pensé eso? Es una combinación de no ver mujeres haciendo esto. Se alimenta en sí mismo... Hay una aceptación de que la femineidad no está unida a la ingeniería".
En el campo de la ingeniería del sonido, en relación con operadores de radio y de emisión de programas, las mujeres representaban en EE.UU., en 2015, el 9,2\%. El porcentaje respecto al decenio comprendido entre 2004 2014 se situaría entre un $7 \%$ y un $16 \%$ de mujeres en este sector laboral (Mathew et al., 2016).

Otros datos nos muestran que aproximadamente un cuarto de las mezcladoras de sonido de la cadena británica BBC son mujeres (Savage, 2012).

Sin embargo, parece que esta discriminación de género es aún más acusada en el desempeño de estas profesiones dentro la propia industria musical. Tal y como recoge la Recording Academy (2021) de EE.UU., al motivar el lanzamiento de su iniciativa Women in the Mix, tan solo el $2 \%$ de las productoras de música y el $3 \%$ de las ingenieras de sonido en la música popular son mujeres.

De hecho, el análisis realizado por Smith et al. en 2020 para evaluar, entre otras variables, la inclusión de mujeres profesionales responsables de la producción musical en 800 éxitos de música popular entre 2012-2019 (usando como medida del éxito de la pieza musical su inclusión anual en las 100 mejores canciones del ranking de Billboard), muestra que por cada mujer productora nos encontramos con 37 hombres o, en otras palabras, de media a lo largo de prácticamente la última década, tan solo el 2,6\% son mujeres productoras ( $v$. tab. 1).

Tabla 1. Porcentajes anvales representación en producción por género

\begin{tabular}{ccccccc} 
& $\mathbf{2 0 1 2}$ & $\mathbf{2 0 1 5}$ & $\mathbf{2 0 1 7}$ & $\mathbf{2 0 1 8}$ & $\mathbf{2 0 1 9}$ & TOTAL \\
\hline \% Hombres & 97,6 & 98,2 & 98,2 & 97,7 & 95 & 97,4 \\
\hline \% Mujeres & 2,4 & 1,8 & 1,8 & 2,3 & 5 & 2,6
\end{tabular}

Fuente: Smith et al., 2020

Otro estudio llevado a cabo por The Music Producers Guild en el Reino Unido, nos señala que menos del $4 \%$ de sus miembros son mujeres (Savage, 2012).
En definitiva, la mayoría de las estimaciones sugieren que las mujeres encargadas del tratamiento técnico del sonido, a las que Elisabet- 
Dobson denomina las de "behind the glass" representan, de media, menos del $5 \%$ en la industria musical (Haruch, 2010; Saxelby, 2014; Dobson, 2018).

A tenor de los datos no es de extrañar que sea común para las mujeres ingenieras de sonido de la industria musical ser la única mujer dentro de los equipos de trabajo (Welch, 2013).

"I was the only woman in the studio where I interned and didn't know women in the field" ${ }^{\prime \prime}$ (Campbell, 2019, como se citó en Gaston-Bird, 2020, p. 76).

"I've only ever worked with one female studio engineer"7 (Levine, como se citó en Savage, 2012, párr. 8).

"I didn't see other people like me doing that"8 (Mari, 2019, como se citó en Gaston-Bird, 2020, p. 74).

\section{Ambientes de trabajo}

La música, como expresión cultural y artística que es, está estrechamente vinculada con el contexto social en el que se desarrolla. La industria musical, como ente en donde mujeres y hombres desempeñan trabajos profesionales ligados de una u otra forma a la música, se encuentra involucrada en los mismos contextos. En estos contextos se observa que, tal y como sostiene Patricia Adkins, "el mundo musical y el sector cultural, como un todo, no están solamente construidos desde una perspectiva masculina, sino que están también constituidos simultáneamente como un medio de perpetua hegemonía cultural masculina y de control social" $(2006$, p. 187).

En sociedad, el éxito profesional es consecuencia de factores muy variados, como pueden ser las propias capacidades individuales, la formación específica y la adquirida a lo largo de ejercicio profesional, el compromiso laboral,

\footnotetext{
5 "Detrás del cristal".

6 "Yo era la única mujer en el estudio donde estaba haciendo prácticas y no conocía a mujeres en este campo".

7 "Solo he trabajado con una ingeniera de estudio".

8 "No vi otras personas como yo haciendo eso".
}

el trabajo duro.... Una persona puede hacerse así misma, pero, en consonancia con lo expuesto por Pamela Laird en su estudio Pull: Networking, and Sucess since Bejamin Franklin, el concepto denominado capital social puede considerarse una piedra angular en el establecimiento, desarrollo y ascenso de las carreras profesionales (Laird, 2007).

El capital social promueve la conexión y la posible conectividad entre individuos. Englobaría todos aquellos recursos sociales que permiten a una persona atraer el respeto y generar confianza de sus pares. El capital social opera a través del establecimiento de redes de trabajo, de mentorías, de que los denominados gatekeepers $^{9}$ te abran puertas y/o de tener modelos en quien inspirarse para querer desempeñar y emular una determinada parcela laboral. Sin embargo, las desigualdades sociales, pueden estar incrustadas en el capital social. Tal y como apunta Robert Putnam (2000, como se citó en Laird, 2007) "norms and networks that serve groups may obstruct others, particularly if the norms are discriminatory or the networks socially segregated"10 (pp. 2-3).

El rol del productor, ingeniero y/o técnico de sonido en la industria de la música ha estado principalmente ligada a la figura de un hombre que se desenvuelve dentro de un ambiente de trabajo a su vez masculinizado y asociado a nociones de control y poder (Ispizua, 2020; Wolfe, 2012). Las oportunidades de aprendizaje, los conocimientos y habilidades técnicas, de desarrollarse profesionalmente necesitan ser llevadas a cabo en el mundo práctico en donde el uso de la tecnología está íntimamente relacionado con la creatividad musical; en un estudio de grabación, en una mesa de mezclas, en el uso de equipos de sonido... Podría ser precisamente a causa de estos ambientes de trabajo dominantemente masculinizados, de estas redes de trabajo socialmente segregadas en función del género, el motivo por el que las mujeres encuentran más dificultades para poder construir una carrera profesional en el campo que estamos analizando.

9 "Guardianes de las puertas".

10 "Las normas y las redes de trabajo que atienden a determinados grupos obstruińan especialmente a otros grupos si esas nomas son discriminatorias o si las redes de trabajo están segregadas socialmente". 
De hecho, esta segregación en función del género en las redes de trabajo se pone de manifiesto en diversos estudios en los que se han analizado las composiciones de las/os miembros de diversas organizaciones que abarcan un amplio espectro de profesionales relacionados específicamente con la tecnología de la música con el objetivo de tratar de conocer la proporción de mujeres y hombres que trabajan en el campo del sonido musical. En concreto, el estudio llevado a cabo por Mathew et al. (2016) se centró en analizar la composición por géneros de dos de las más significativas organizaciones con diversas sedes internacionales que abarcan un gran espectro de profesiones relacionadas con la tecnología del sonido musical: la International Computer Music Asociation (ICMA) y la Audio Engineering Society (AES).

Los menores porcentajes de mujeres que componen tanto una como otra organización revelan que existe una brecha de género en ellas, ya que, si bien se observa un ligero incremento en el número de mujeres que han ido incorporándose a ellas a lo largo del último decenio, los niveles permanecen aún muy bajos en comparación con el número de hombres; con valores oscilantes entre el 14,5\%-19,6\% a lo largo de una década para ICMA a tan solo el 7\% en 2016 para AES. Además, una menor representación femenina en la organización de AES, más íntimamente ligada con la tecnología del sonido, con respecto a la ICMA, que abarca campos algo más generales de la música, nos puede ilustrar algo más acerca de la infrarrepresentación de las mujeres en los campos específicamente relacionados con las áreas científico-tecnológicas de la industria musical.

En el estado español, la actual Junta Directiva de la sección española de AES, AES Spain, está compuesta por un 22,2\% de mujeres, estando presidida por una mujer (Audio Engineering Society [AES], 2021 b). La representación femenina en la sección española es, sin embargo, 9 puntos inferior que la representación actual en la Junta Directiva de AES central que es del 31,8\% (AES, 2021a). No obstante, dicha Junta Directiva central no tuvo una mujer en la misma hasta 1984 (36 años después de su fundación) y solamente ha estado presidida por cinco mujeres a lo largo de sus más de 70 años de existencia (Gaston-Bird, 2020).

\section{Redes de trabajo}

Para tratar de construir espacios menos hostiles de trabajo es por lo que las mujeres se han visto en la necesidad de trazar sus propias redes de trabajo, organizándose para generar sinergias que promuevan la eliminación de la discriminación de género y alcancen una igualdad efectiva, en donde tienen su representatividad y sus voces sean escuchadas. En este sentido, hemos asistimos a la creación de muy diversas asociaciones y organizaciones vinculadas con numerosos sectores de la industria musical, que a lo largo de todo el mundo se han constituido para ser plataformas donde las mujeres pueden mostrar su trabajo, compartir sus logros e inquietudes, sean lugares de encuentro profesional, generen oportunidades de formación y promoción profesional, y actúen como centros de inspiración para las próximas generaciones de jóvenes interesadas en desarrollase laboralmente en alguno de los campos profesionales que conforman la industria musical en general, pudiendo destacar también las que específicamente promueven el desarrollo de las carreras profesionales de mujeres en el campo del tratamiento del sonido musical.

Estas organizaciones no están articuladas como vehículos donde las mujeres relacionadas con el tratamiento del sonido en la música expresen sus quejas, sino que están enfocadas en servir de herramientas para apoyarse y conectar entre ellas en un campo ampliamente dominado por hombres. Además, actúan como centros de visibilización donde las jóvenes pueden encontrar modelos donde inspirarse en las mujeres veteranas que han desarrollado su carrera dentro de los campos técnicos del sonido (Welch, 2013), además de proporcionar entornos donde las mujeres puedan experimentar con las técnicas del sonido musical sin ser continuamente evaluadas por sus pares masculinos o donde sus habilidades y cualidades técnicas no sean escudriñadas simplemente por el mero hecho de ser mujer.

"It's really important to have support and having women that are veterans in this industry, is great to provide as a role model"11 (Keyes, como se citó en Welch, 2013, párr. 6).

"It's nice to have a place where women can

11 "Es realmente importante tener apoyo y tener mujeres veteranas en esta industria, es bueno para proporcionar modelos". 
be there to support each other with specific issues"12 (Sabolchick, como se citó en Welch, 2013, párr. 7).

We have to be in an environment which is lower risk, which -in this case- means not being the only woman in a world which associates masculinity and technology; to remove that risk to be in a community of peers; and to have a chance to put your hands on and use equipment. To make mistakes and learn from that. ${ }^{13}$ (Dobson, 2019, como se citó en Gaston-Bird, 2020, p. 83).

El trabajo desarrollado por las organizaciones y asociaciones ha contribuido a que poco a poco se pueda encontrar un número cada vez más creciente de mujeres involucradas en el mundo del sonido musical (Gaston-Bird, 2020), aunque su visibilización en los entornos laborales aún diste mucho de estar en igualdad con la de sus compañeros masculinos.

Dentro de las 66 organizaciones que están actualmente trabajando, tanto para proveer de recursos necesarios, como para elevar la visibilidad de las mujeres dentro del campo del tratamiento técnico del sonido, que ha compilado Elisabeth Dobson (Gaston-Bird, 2020, pp. 299-306), podríamos mencionar algunas más significativas y orientadas a la industria musical, (Recording Academy, 2021; Smith ef al., 2020; Command, 2017) como por ejemplo, Women's Audio Mission, SoundGirls.org, Female Frequency, Women In Sound, Women On Sound y Yorkshire Sound Women Network, entre otras. En el estado español se están empezando a crear iniciativas asociativas en este sentido.

\section{CONCLUSIONES}

Al constituirse la industria de la música en uno de los sectores más potentes y de mayor crecimiento dentro del conjunto de las industrias culturales, es por lo que el estudio de la misma presenta una gran importancia para identificar los estereotipos de género o sesgos androcén-

12 "Es bueno tener un lugar donde las mujeres puedan estar alli para apoyarse mutuamente en problemas espećficos".

13 "Tenemos que estar en un medio que sea menos amiesgado, en el que no seas la única mujer en un mundo que asocia masculinidad con tecnología; para eliminar el riesgo de estar en una comunidad de compañeros; y para tener una oportunidad de poner tus manos en los equipos y usarlos tricos para en su caso ponerlos en evidencia y contribuir al proyecto de construcción de una sociedad más igualitaria (Martínez Berriel, 2011; Marinas, 2019).

El cuestionar el androcentrismo en puestos de producción e ingeniería del sonido en la industria musical puede actuar como un motor de cambio social, ya que su repercusión abarcaría más allá de las salas de conciertos o de los teatros, puesto que la presencia de a música está en, prácticamente, todas nuestras acciones cotidianas.

La menor representación femenina en los campos profesionales de la industria musical relacionados con el tratamiento técnico del sonido (personas especialistas en sonido -ingenieras/os y técnicas/os de sonido-, las personas encargadas de la producción musical) con respecto a la ya de por sí escasa presencia de mujeres en otros campos profesionales de la industria de la música, tendría su causa en un mayor sesgo androcéntrico (She is the Music, s.f.; Marinas, 2019; Smith et al., 2020) en relación con varios factores entre los que se destacan:

- La tradicional existencia de una segregación en el empleo y un desequilibrio en las titulaciones científico-tecnológicas para las mujeres. Así, las mujeres especialistas en sonido no cumplirían con un determinado "canon" como afirmaba Citron (1993); en este caso el canon establecido del campo técnico dominado por los hombres.

- Las personas especialistas del sonido al utilizar las distintas herramientas a su alcance (estudios de grabación, territorios propios de la acústica musical, mesas de mezclas...) junto con sus respectivos bagajes culturales, estarían interpretando en cada ocasión una obra musical distinta; les imprimirían a las obras una impronta particular. Si esto fuera así, las mujeres profesionales del sonido tampoco responderían al canon de la creatividad, también enunciado por Citron (1993), puesto que la creatividad, y no solo en la música, parece ser una actividad que está casi exclusivamente en manos masculinas.

- Redes de trabajo en las que se perpetúan ambientes laborales hostiles para 
el desarrollo profesional de las mujeres en el campo de la tecnología sonido. Las mujeres profesionales de la tecnología del sonido al potenciar la creación de asociaciones y organizaciones en donde entre sus objetivos fundamentales estuviesen el proveer de una comunidad en la que estas profesionales se apoyasen, se aconsejasen y fuera motor de empoderamiento e inspiración para ellas, el fomentar campos de educación y entrenamiento para niñas y mujeres que desean aprender las disciplinas relacionadas con las técnicas de producción y grabación de sonido y el incrementar la visibilización las mujeres que desempeñan su labor profesional en esos campos, estarían colaborando a construir un nuevo capital social.

Es por todo ello por lo que las mujeres que se desarrollan laboralmente en este campo podrían estar contribuyendo a la elaboración de identidades femeninas no tradicionales (Green, 2001). Es decir, que al cuestionar los diferentes cánones y al establecer sus propias redes de trabajo estarían poniendo en entredicho el relato establecido y estarían abriendo la oportunidad a enunciar nuevas narrativas.

Según Small (1998) la música solo tiene sentido cuando se interpreta en directo o a través de una grabación, pero esta no solo es el fruto del trabajo de una única persona, sino que es el resultado de la interactuación de todas las personas que tienen una $u$ otra presencia en la misma; es lo que Small denominó musicking ${ }^{14}$. Tanto en el caso de la música grabada como de la música en directo, en la industria musical es necesario una persona especialista de sonido para el acto musical y podría ocurrir que las mujeres dotaran a su labor profesional, consciente o inconscientemente, de una nueva significación de género (Viñuela, 2003).

14 Término que "engloba a todas las personas que forman parte del acto musical. Desde el personal de limpieza de la sala donde se realiza el concierto, a los intérpretes, al público, a quienes venden las entradas. ..." (Soler, 2018, p. 95).

\section{REFERENCIAS BIBLIOGRÁFICAS}

Adkins, P. (2006). El futuro de la música-mujeres en la música. La creación y el apoyo de una Red Global. Mujeres y mercado musical. Las mejores piezas del género dominante y su autorización. Papeles del Festival de Música Española de Cádiz, 2, 181-200.

Asociación de Promotores Musicales. (2020). Anuario de la música en vivo 2020. Especial XX aniversario. http://anuario.apmusicales. $\mathrm{com} /$

Audio Engineering Society. (2021a). AES Officer and Committees. Recuperado el 03 de enero de 2021 de https://www.aes.org/ about/leadership/

Audio Engineering Society. (2021b). Spanish AES Section. Recuperado el 03 de enero de 2021 de https://www.aes.org/sections/ view.cfm? section $=106$

Banks, M., y Milestone, K. (2011). Individualization, gender and cultural work. Gender Work and Organization, 18(1), 73-89.

Bilbao, M. (2015). Música, cultura popular y capitalismo. El género de la música. Viento Sur, 141, 82-88.

Cabedo, A. (2009). Música, género y paz: Anotaciones a partir de los estudios musicológicos feministas. Revista Tiempo de paz, 95, 86-92. http://hdl.handle. net/10234/25183

Cascudo García-Villaraco, T. (1998). Los trabajos de Penélope musicóloga: musicología y feminismo entre 1974 y 1994. En Manchado, M., (ed.): Música y mujeres: Género y poder. Madrid: Horas y Horas.

Citron, M. J. (1993). Gender and the Musical Canon. Cambridge: Cambridge University Press.

Command, Sarah (18 de diciembre de 2017). 5 Women In Music Organizations You Should Know About. The Women's International Music Network. https://www.thewimn. com/5-women-music-organizations-know/

Cruz, A. (2019). La brecha de género en la música profesional. Las mujeres son mayoría en los conservatorios, pero solo 3 de cada 10 forman parte de una orquesta profesio- 
nal. http://brechageneromusicaprofesional. aranzazucruz.com/

Die Goyanes, A. (1998). Mujeres en la música. En Manchado, M., (ed.): Música y mujeres: Género y poder. Madrid: Horas y Horas.

Dobson, E. (2018). Digital Audio Ecofeminism (DA'EF). The Glocal Impact of All-Female Communities on Learning and Sound Creativities. En L.R. de Bruin, P. Burnard y S. Davis (Eds.), Creativities in Arts, Education, Research and Practice (pp. 201-220). Leiden, Holanda: Brill|Sense.

Fuente, U. (8 de septiembre de 2020). El "streaming" aumenta los beneficios de la industria musical durante la pandemia. La Razón. https://www.larazon.es/cultura/20200908/ cgaay34k45f43aqbbmfhx 5 dfoy. html

Gaston-Bird, Leslie. (2020). Women in Audio. New York and London: Routledge, Taylor\&Francis Group.

González González, J. M. (1999). La situación de las mujeres en los estudios universitarios técnicos. Sevilla, Málaga: Instituto Andaluz de la Mujer.

Green, L. (2001). Música, género y educación. Madrid: Morata.

Haruch, S. (3 de junio de 2010). Women account for less than 5 percent of producers and engineers-but maybe not for long. Nashville Scene News. https://www.nashvillescene.com/news/article/13034234/ women-account-for-less-than-5-percent-ofproducers-and-engineers-but-maybe-notfor-long

Hopkins, E. y Berkers, P. (2019). Engineering a place for women: Gendered experiences of the music technology classroom. En S. Raine y C. Strong (Eds.) Towards gender equality in the music industry: Education, practice and strategies for change (pp. 45-88). New York: Bloomsbury.

Ispizua, A. (2020). Situación de mujeres y hombres en la industria de la música en la CAE. San Sebastián, Vitoria: Servicio Central de Publicaciones del Gobierno Vasco.

Keychange. (2018). Foreword from Vanessa Reed PRS Foundation, CEO. [Booklet de- signed by lan Robson at Suit Suit]. PRS Foundation. Copia en posesión de la autora.

Laird, Pamela (2007). Pull: Networking and Success since Benjamin Franklin. Cambridge, Massachusetts: Harvard University Press.

Live DMA. (2020). La encuesta: salas de conciertos y clubs en Europa-hechos y cifras. https://www.live-dma.eu/wp-content/uploads/2021/01/survey_esp_BD-1.pdf

Lores, A. (11 de mayo de 2017). ¿̇Por qué todas las mujeres de la música trabajan en comunicación (y no son directivas)? El País. https://elpais.com/elpais/2017/05/10/ tentaciones/1494429397_297078.html

Manchado, M. (1998). Música y mujeres: Género y poder. Madrid: Horas y Horas.

Mañó, V. y Bori, J. (2016). Así funciona el negocio de la música. Valencia: Editorial Círculo Rojo.

Marinas, L. (2019). Mujeres en la industria musical: políticas públicas para la participación, la visibilidad y la igualdad. Estudios de Progreso. Fundación Alternativas, 98/2019. https://www.fundacionalternativas.org/ public/storage/estudios documentos archivos/bbdb5604d8aē ld4d3dc456e0c58e5a20.pdf

Martínez Berriel, S. (2011). El género de la música en la cultura global. TRANS. Revista Transcultural de Música, 15, 1-17. https:// www.sibetrans.com/trans/articulo/370/elgenero-de-la-musica-en-la-cultura-global

Martínez Escribano, L. M. (2011). Sexismo en la música pop española: Análisis desde la autoría e interpretación. [Ponencia] Investigación y Género, Logros y Retos: III Congreso Universitario Nacional Investigación y Género, [Libro De Actas] (pp. 1214-1223).

Mathew, M.; Grossman, J.; Andreopoulou, A. (2016). Women in Audio: contributions and challenges in music technology and production. 147 st Convention Audio Engineering Society, [Paper 9673]. Los Ángeles, USA.

Mujeres y Música. (2021). La presencia de las mujeres en los festivales 2019. Recuperado el 07 de enero de 2021 de http://mu- 
jeresymusica.com/presencia-de-las-mujeres-en-los-festivales-en-2019/

Piñero, C. C. (2003). La transgresión de Euterpe: Música y género. Dossiers Feministes, 7, 45-64.

Prior, B., Barra, E., Kramer, S. (2018). Women in the U.S. Music Industry: Obstacles and Opportunities. Berklee Institute for Creative Entrepreneurship.

PromociónMusical.es. (s.f.). Industria Musical: Qué es, Historia, Estructura y Modelo de Negocio. Recuperado el 20 de enero de 2021 de https://promocionmusical.es/industria-musical-que-es-historia-modelo-negocio/

Quiñones, L. (11 de febrero de 2019). Mujeres en la música, silenciadas por la desigualdad de género. Noticias ONU. https:// news.un.org/es/story/2019/02/1450871

Ramos López, P. (2003). Feminismo y música: introducción crítica. Madrid: Narcea, S.A. Ediciones.

Ramos López, P. (2010). Luces y sombras en los estudios sobre las mujeres y la música. Revista Musical Chilena, 64(213), 7-25.

Ramos López, P. (2013). Una historia particular de la música: La contribución de las mujeres. Brocar. Cuadernos De Investigación Histórica, 37, 207-224.

Recording Academy. (2021). Women in the Mix: Producer \& Engineering Inclusion Initiative. Recuperado el 07 de enero de 2021 de https://www.grammy.com/recording-academy/women-mix-producer-engineering-inclusion-initiative

Savage, M. (29 de agosto de 2012). Why are female record producers so rare? BBC News. https://www.bbc.com/news/entertainment-arts-19284058

Saxelby, R. (30 de octubre de 2014). 13 women on How to change male-dominated studio culture. The Fader. https://www.thefader. com/2014/10/30/why-arent-more-women-becoming-music-producers

She is the music (s.f.). Mission. https://sheisthemusic.org/mission/
Small, C. (1998). Musicking: The meanings of performing and listening. USA: University Press of New England.

Smith, S. L., Pieper, K., Clark, H., Case, A. y Choueiti, M. (2020). Inclusion in the Recording Studio? Gender and Race/Ethnicity of Artists, Songwriters \& Producers across 800 Popular Songs from 2012-2019. http:// assets.uscannenberg.org/docs/aii-inclusion-recording-studio-202001 17.pdf

Soler, S. (2018). Cuestiones de género: mujeres en la historia de la música. ARTSEDUCA, 19, 84-101. DOl - http://dx.doi. org/10.6035/Artseduca.2018.19.5

Viñuela, L. (2003). La construcción de las identidades de género en la música popular. Dossiers Feministes, 7, 11-32.

Viñuela, L. (2004). La perspectiva de género y la música popular: Dos nuevos retos para musicología. Oviedo: Krk.

Welch, K. (8 de octubre de 2013). Veteran Engineers Create SoundGirls. Org. ProSound News. https://www. prosoundnetwork.com/ business/veteran-engineers-create-soundgirlsorg

Wikström, P. (2014). La industria musical en una era de distribución digital. En BBVA, C@ambio: 19 ensayos fundamentales sobre cómo internet está cambiando nuestras vidas (pp. 1-23). BBVAOpenMind. https:// www.bbvaopenmind.com/wp-content/uploads/201 4/04/BBVA-OpenMind-La-industria-musical-en-una-era-de-distribucion-digital-Patrik-Wikstrom.pdf.pdf

Wolfe, P. (2012). A studio of one's own: music production, technology and gender. Journal on the Art of Record Production, 7. https:// www.arpjournal.com/asarpwp/a-studio-of-one\%e2\%80\%99s-own-music-production-technology-and-gender/

Zavala, M. (2012). Música y Género. En Suárez Ojeda, M., (ed.): Género y Mujer desde una perspectiva multidisciplinar. Madrid: Editorial Fundamentos. 
